PROCEEDINGS OF THE

AMERICAN MATHEMATICAL SOCIETY

Volume 135, Number 7, July 2007, Pages 1969-1975

S 0002-9939(07)08678-9

Article electronically published on February 28, 2007

\title{
CENTRALIZERS IN FREE POISSON ALGEBRAS
}

\author{
LEONID MAKAR-LIMANOV AND UALBAI UMIRBAEV
}

(Communicated by Martin Lorenz)

\begin{abstract}
We prove an analog of the Bergman Centralizer Theorem for free Poisson algebras over an arbitrary field of characteristic 0 . Some open problems are formulated.
\end{abstract}

\section{INTRODUCTION}

There are many results, some of them quite deep, known about the structure of polynomial algebras, free associative algebras, and free Lie algebras. Although free Poisson algebras are very closely connected with these algebras, only a few results are known about them up to now.

Free Poisson algebras are sometimes useful in research of polynomial algebras. For example the free Poisson algebra of rank three and Poisson brackets were used recently in [21, 15, 16, 17] to prove that the Nagata automorphism (see [13])

$$
\sigma=\left(x+\left(x^{2}-y z\right) z, y+2\left(x^{2}-y z\right) x+\left(x^{2}-y z\right)^{2} z, z\right)
$$

of the polynomial algebra $F[x, y, z]$ over a field $F$ of characteristic 0 is wild.

Among very few works devoted exclusively to Poisson algebras let us mention [6, where all homogeneous quadratic Poisson structures on $F[x, y, z]$ are described, and 14, where algebraic quantizations of free Poisson algebras are constructed.

One of the fundamental results about free associative algebras is the Bergman Centralizer Theorem (see 11) which says that the centralizer of any nonconstant element is a polynomial algebra on a single variable. This theorem plays a crucial role in the study of algorithmic and combinatorial questions.

An analog of this theorem for polynomial algebras was proved in [16]; see also 23. In the case of free Poisson algebras the question about the validity of an analog of the Bergman Centralizer Altheorem was open and was formulated in [16, Problem 1].

In Section 2 of this paper we prove that in the characteristic zero case the centralizer of any nonconstant element of a free Poisson algebra is a polynomial algebra on a single variable. Although Bergman's Theorem is characteristic-free, in our setting we should assume that the characteristic of the ground field $F$ is zero.

Received by the editors May 17, 2005 and, in revised form, February 20, 2006.

2000 Mathematics Subject Classification. Primary 17B63, 17A50; Secondary 17B40, 17A36, $16 \mathrm{~S} 10$.

Key words and phrases. Poisson algebras, centralizers, subalgebras.

The first author was supported by an NSA grant.

(C)2007 American Mathematical Society Reverts to public domain 28 years from publication 
When the characteristic is finite, free Poisson algebras have large centers and the centralizers are much larger than in the zero characteristic case.

Some open problems are formulated in Section 3.

\section{Centralizers in free Poisson algebras}

First, we present a proof of one useful lemma about polynomial algebras.

Let $A=F\left[z_{1}, \ldots, z_{n}, \ldots\right]$ be a polynomial algebra in any number of variables over a field $F$. Let $\operatorname{deg}$ be a weight degree function on $A$, i.e. $\operatorname{deg}\left(z_{i}\right)=d_{i}$, where $d_{i}$ are nonnegative integers. Denote by $\bar{a}$ the highest homogeneous part of $a \in A$, i.e. $\bar{a}=\sum_{i} \alpha_{i} a_{i}$, where $a_{i}$ are the monomials of $a$ of the maximal possible degree and $\alpha_{i} \in F$ are the corresponding coefficients, so that $a=\bar{a}+r$ where $\operatorname{deg}(r)<\operatorname{deg}(a)$. Call an element $b$ homogeneous if $b=\bar{b}$.

For a given $f \in A$, let $C l(f)$ denote the set of all elements of $A$ which are algebraically dependent with $f$. By a theorem of A. Zaks [23] $C l(f)=F[t]$ for some $t \in A$. So elements $f, g \in A$ are algebraically dependent iff there exists $t \in A$ such that $f, g \in F[t]$. If $f$ is homogeneous and $\operatorname{deg}(f)>0$, then it is easy to see that $t$ is also homogeneous and $\operatorname{deg}(t)>0$. Consequently, if $f, g \in A$ are algebraically dependent homogeneous elements and $\operatorname{deg}(f)>0$, then there exists a homogeneous element $t$ such that $f=\alpha t^{r}, g=\beta t^{k}$, where $\alpha, \beta \in F$ and $r, k$ are nonnegative integers.

For the reader's convenience we present a proof of the next lemma, though similar lemmas are already proved in the literature, e.g. see 9 and [16. The idea of the proof can be found in $[7$.

Lemma 1. Let $f, g \in A$ be two algebraically independent elements. There exists an element $h \in F[f, g]$ such that $\bar{f}$ and $\bar{h}$ are algebraically independent.

Proof. Suppose that $\operatorname{deg}(f)=0$. Then $f=\bar{f}$. If $\operatorname{deg}(g)>0$, then $f$ and $\bar{g}$ are algebraically independent. On the other hand if $\operatorname{deg}(g)=0$, then $\bar{g}=g$. In both cases we can take $h=g$.

Now assume that $\operatorname{deg}(f)>0$. Denote $F[f, g]$ by $B$. Let $B_{n}$ be the subset of $B$ that consists of the elements of the form $q(f, g)$ where the standard (total) degree of the polynomial $q$ is $\leq n$. Then $B_{n}$ is a linear space over $F$ and the dimension $d_{n}$ of $B_{n}$ is $O\left(n^{2}\right)$ since $f$ and $g$ are algebraically independent $\left(d_{n}=\left(\begin{array}{c}n+2 \\ 2\end{array}\right)\right)$. Assume that $\bar{b}$ and $\bar{f}$ are algebraically dependent for any $b \in B$. To get a contradiction we show that under this assumption $d_{n}$ cannot grow as $O\left(n^{2}\right)$.

Indeed, we know that there exists a homogeneous element $c \in A$ such that $C l(\bar{f})=F[c]$ and $\operatorname{deg}(c)>0$. Then $\bar{b}=\lambda_{b} c^{z_{b}}$.

Consider the set $D_{n}$ of all possible degrees of the elements of $B_{n}$. It is clear that $\operatorname{deg}(q(f, g)) \leq n(\operatorname{deg}(f)+\operatorname{deg}(g))$ if the standard degree of $q$ is $\leq n$. So $D_{n}$ has at most $n(\operatorname{deg}(f)+\operatorname{deg}(g))+1$ elements. For any degree present in $D_{n}$ choose an element $b_{i} \in B_{n}$ with this degree. Since under our assumption $\overline{b_{i}}$ is determined by $\operatorname{deg}\left(b_{i}\right)$ up to multiplication by an element of $F$, we can conclude that $\operatorname{dim}\left(B_{n}\right) \leq n(\operatorname{deg}(f)+\operatorname{deg}(g))+1$ and grows linearly.

Now, we recall the definition of Poisson algebras. A vector space $B$ over a field $F$ endowed with two bilinear operations $x \cdot y$ (a multiplication) and $[x, y]$ (a Poisson bracket) is called a Poisson algebra if $B$ is a commutative associative algebra under $x \cdot y, B$ is a Lie algebra under $[x, y]$, and $B$ satisfies the following identity (the 
Leibniz identity):

$$
[x \cdot y, z]=[x, z] \cdot y+x \cdot[y, z] .
$$

Of course, the Leibniz identity just says that for every $x \in B$ the map

$$
a d x: B \longrightarrow B, \quad(y \mapsto[y, x]),
$$

is a derivation of $B$ as an associative algebra.

An important class of Poisson algebras is given by the following construction. Let $L$ be a Lie algebra with a linear basis $l_{1}, l_{2}, \ldots, l_{k}, \ldots$ over $F$. Denote by $P(L)$ the polynomial algebra on the variables $l_{1}, l_{2}, \ldots, l_{k}, \ldots$ Using the Leibniz identity we can uniquely extend the Lie bracket $[x, y]$ of $L$ to a Poisson bracket $[x, y]$ on $P(L)$, and $P(L)$ becomes a Poisson algebra. This algebra is called a Poisson-Lie algebra.

We will say that elements $f$ and $g$ of $P(L)$ are algebraically dependent if they are algebraically dependent as polynomials.

Lemma 2. If $f, g \in P(L)$ are algebraically dependent, then $[f, g]=0$.

Proof. The proof is quite standard. Take an algebraic dependence $h$ of $f$ and $g$ with, say, minimal possible degree relative to $g$. Then $0=[f, h(f, g)]=[f, g] \frac{\partial h}{\partial g}(f, g)$, and since $\frac{\partial h}{\partial g}(f, g) \neq 0$ we see that $[f, g]=0$.

Remark. Although the statement of this lemma is correct when the characteristic is finite, this proof works only for the characteristic zero case.

If $f \in A=F\left[z_{1}, \ldots, z_{n}, \ldots\right]$, then we know that $C l(f)=F[t]$ for some $t \in A$. So in order to prove "the centralizer" theorem for a Poisson-Lie algebra $P$ it is sufficient to check that commuting elements of $P$ are also algebraically dependent. Of course this cannot be done without additional restrictions. As we already mentioned the characteristic is critical. It is also clear that the structure of $L$ plays a role: if e.g. $L$ is abelian, then $P(L)$ is commutative, etc.

From now on let $L$ be a free Lie algebra with free (Lie) generators $x_{1}, x_{2}, \ldots, x_{n}$. It is well known (see, for example [14) that in this case $P(L)$ is the free Poisson algebra on the same set of generators, and we denote this algebra by $P=$ $P\left\langle x_{1}, x_{2}, \ldots, x_{n}\right\rangle$. By deg we denote the standard degree function of the algebra $P$, i.e. $\operatorname{deg}\left(x_{i}\right)=1$, where $1 \leq i \leq n$. For every element $f \in P$ the highest homogeneous part $\bar{f}$ can be defined in the ordinary way. Note that

$$
\overline{f g}=\bar{f} \bar{g}, \operatorname{deg}[f, g] \leq \operatorname{deg} f+\operatorname{deg} g .
$$

Let us choose a homogeneous basis

$$
x_{1}, x_{2}, \ldots, x_{n},\left[x_{1}, x_{2}\right], \ldots,\left[x_{1}, x_{n}\right], \ldots,\left[x_{n-1}, x_{n}\right],\left[\left[x_{1}, x_{2}\right], x_{3}\right], \ldots
$$

of $L$. The algebra $P=P\left\langle x_{1}, x_{2}, \ldots, x_{n}\right\rangle$ coincides with the algebra of polynomials on these elements, and the degree function deg can be considered as a weight degree function on this polynomial algebra, where $\operatorname{deg}\left[\ldots\left[x_{i_{1}}, x_{i_{2}}\right], \ldots, x_{i_{k}}\right]=k$ if $\left[\ldots\left[x_{i_{1}}, x_{i_{2}}\right], \ldots, x_{i_{k}}\right] \neq 0$.

For every $f \in P$ the set of elements

$$
\mathcal{C}(f)=\{g \in P \mid[f, g]=0\}
$$

is called the centralizer of $f$. It follows immediately from the Leibniz and Jacobi identities that $\mathcal{C}(f)$ is a subalgebra of $P$. 
Take $f \in P \backslash F$. We would like to prove that any $g \in \mathcal{C}(f)$ is algebraically dependent with $f$. So let us assume that some $g \in \mathcal{C}$ is not algebraically dependent with $f$. By Lemma 1 there exists a polynomial $h$ in $f$ and $g, h \in F[f, g]$, such that $\bar{f}$ and $\bar{h}$ are also algebraically independent. Since $[f, h]=0$ and consequently $[\bar{f}, \bar{h}]=$ 0 we may assume that there is a pair of homogeneous algebraically independent commuting elements.

There is another natural degree function on $P$, just the total degree on $P$ as a polynomial ring, where the degree is one for all elements of the homogeneous basis of $L$. Denote it by pdeg and observe that pdeg $[a, b]=\operatorname{pdeg} a+\operatorname{pdeg} b-1$ for any phomogeneous $a, b \in P$ if $[a, b] \neq 0$. Therefore if $[f, g]=0$ and $\widetilde{a}$ denotes the highest p-homogeneous form of $a$, then $[\tilde{f}, \widetilde{g}]=0$ as well. Of course, Lemma 1 implies that if $f$ and $g$ are algebraically independent, then there exists an $h \in F[f, g]$ for which $\widetilde{f}$ and $\widetilde{h}$ are algebraically independent. So if we start with a pair of algebraically independent commuting elements we can obtain a pair of algebraically independent commuting elements which are homogeneous and p-homogeneous. Let us call such elements bi-homogeneous.

Lemma 3. Let $f$ and $g$ be bi-homogeneous elements of $P \backslash F$ such that $[f, g]=0$. Then there exists $a \in P$ such that $f, g \in F[a]$, i.e. $f$ and $g$ are algebraically dependent.

Proof. Let us denote the elements of the basis

$$
x_{1}, x_{2}, \ldots, x_{n},\left[x_{1}, x_{2}\right], \ldots,\left[x_{1}, x_{n}\right], \ldots,\left[x_{n-1}, x_{n}\right],\left[\left[x_{1}, x_{2}\right], x_{3}\right], \ldots
$$

by $e_{1}, e_{2}, \ldots, e_{n}, \ldots$ Put $e_{i}<e_{j}$ if $i<j$. It is clear that $\left[e_{m}, e_{n}\right]$ is a bihomogeneous element of $P$ and that $\operatorname{deg}\left[e_{m}, e_{n}\right]=\operatorname{deg} e_{m}+\operatorname{deg} e_{n}$ if $m \neq n$. So if $m<n$, then $\left[e_{m}, e_{n}\right]$ is a linear combination of $\left\{e_{i}\right\}$ where all $i>n$.

For every element $f \in P$ put

$$
S(f)=\left\{e_{i_{1}}, e_{i_{2}}, \ldots, e_{i_{k}}\right\}
$$

if $f \in F[S(f)]$ and $f \notin F\left[S(f) \backslash\left\{e_{i_{j}}\right\}\right]$, where $1 \leq j \leq k$.

Assume now that $f$ and $g$ are bi-homogeneous algebraically independent elements of $P$ such that $[f, g]=0$ and the number of elements $|S(f)|$ in $S(f)$ is minimal possible; of course then $|S(f)| \leq|S(g)|$.

Let $x$ be the minimal element of $S(f)$. We can write

$$
f=f_{0}+f_{1} x+\ldots+f_{m} x^{m},
$$

where $f_{m} \neq 0, m>0$, and $x \notin S\left(f_{i}\right)$ for all $i$ and

$$
g=g_{0}+g_{1} x+\ldots+g_{n} x^{n}
$$

where $g_{n} \neq 0$ and $x \notin S\left(g_{i}\right)$ for all $i$. We can assume that $f_{m} x^{m}$ and $g_{n} x^{n}$ are algebraically independent. Indeed, we introduce a new degree function on $P$ (considered as a polynomial algebra) which is induced by $\operatorname{deg} x=1$ and $\operatorname{deg} e_{i}=0$ if $e_{i} \neq x$, and use Lemma 1 to find $h \in F[f, g]$ such that the corresponding highest homogeneous parts $f_{m} x^{m}$ and $h_{k} x^{k}$ are algebraically independent.

Now,

$$
[f, g]=\sum_{i \leq m+n} d_{i} x^{i}=0
$$

where $x \notin S\left(d_{i}\right)$ for all $i$. Consequently, $d_{i}=0$. So $d_{m+n}=\left[f_{m}, g_{n}\right]=0$. Since $\left|S\left(f_{m}\right)\right|<|S(f)|$ we can conclude that $f_{m}$ and $g_{n}$ are algebraically dependent. 
They are also bi-homogeneous polynomials, hence up to multiplication by constants $f_{m}=a^{s}$ and $g_{n}=a^{t}$ for some $a \in P$. Therefore

$$
d_{m+n-1}=\left[t a^{t-1} f_{m-1}-s a^{s-1} g_{n-1}+(m t-n s) a^{s+t-1} x, a\right]=0 .
$$

Since $f_{m} x^{m}$ and $g_{n} x^{n}$ are algebraically independent, $a \notin F$ and $m t-n s \neq 0$. So $a$ and $t a^{t-1} f_{m-1}-s a^{s-1} g_{n-1}+(m t-n s) a^{s+t-1} x$ are algebraically independent since $S(a) \not \ngtr x$. If $s>0$, then $|S(a)|=\left|S\left(f_{m}\right)\right|<|S(f)|$, and we would have a contradiction with our choice of $f$. So $s=0$ and $\left[f_{m-1}+m x, a\right]=0$. Since $\left|S\left(f_{m-1}+m x\right)\right| \leq|S(f)|$ we can assume that $f=f_{m-1}+m x$. Therefore $f$ is a linear polynomial. Since $a \in \mathcal{C}(f)$, choose a bi-homogeneous $g \in \mathcal{C}(f) \backslash F$ such that $S(g) \not \supset x$ and $|S(g)|$ is minimal possible. The elements $f$ and $g$ are algebraically independent since $S(f) \ni x$ and $S(g) \not \supset x$.

Let $y$ be the minimal element of $S(g)$. Then

$$
f=f_{0}+f_{1} y,
$$

where $f_{1} \in F$ and $y \notin S\left(f_{0}\right)$, and

$$
g=g_{0}+g_{1} y+\ldots+g_{n} y^{n},
$$

where $g_{n} \neq 0, n>0$, and $y \notin S\left(g_{i}\right)$ for all $i$. Of course $y \neq x$ since $S(g) \not \supset x$. As above, $[f, g]=0$ implies $\left[f_{0}+f_{1} y, g_{n}\right]=0$. Since $|S(g)|>\left|S\left(g_{n}\right)\right|$ and $S\left(g_{n}\right) \not \supset x$ we can conclude that up to multiplication by a constant $g_{n}=\left(f_{0}+f_{1} y\right)^{t}$. Hence $t=0$, since otherwise $S\left(g_{n}\right)=S(f) \ni x$. So $g_{n} \in F$. Next, $\left[f_{0}, g_{n-1}\right]+n g_{n}\left[f_{0}, y\right]+$ $f_{1}\left[y, g_{n-1}\right]=0$. But $\left[f_{0}, g_{n-1}\right]+n g_{n}\left[f_{0}, y\right]+f_{1}\left[y, g_{n-1}\right]=\left[f_{0}+f_{1} y, g_{n-1}+n g_{n} y\right]$. Both $f_{0}+f_{1} y$ and $g_{n-1}+n g_{n} y$ are linear polynomials. Therefore we can treat them as elements of $L$. These elements are linearly independent since $x \in S\left(f_{0}+f_{1} y\right)$ and $x \notin S\left(g_{n-1}+n g_{n} y\right)$. Therefore, they cannot commute because $L$ is a free Lie algebra. The lemma is proved.

Lemmas 2 and 3 give us the main result.

Theorem 1. In free Poisson algebras over a field of characteristic 0 the centralizer of every nonconstant element is a polynomial algebra on a single variable.

\section{Problems}

Let $P\left\langle x_{1}, x_{2}, \ldots, x_{n}\right\rangle$ be a free Poisson algebra over a field $F$ in variables $x_{1}, x_{2}$, $\ldots, x_{n}$. For every $f \in P\left\langle x_{1}, x_{2}, \ldots, x_{n}\right\rangle$ we denote by $(f)$ the two-sided ideal of $P\left\langle x_{1}, x_{2}, \ldots, x_{n}\right\rangle$ generated by $f$.

Problem 1 (Freiheitssatz). If $f \in P\left\langle x_{1}, x_{2}, \ldots, x_{n}\right\rangle$ and $f \notin P\left\langle x_{1}, x_{2}, \ldots, x_{n-1}\right\rangle$, then is it true that $(f) \cap P\left\langle x_{1}, x_{2}, \ldots, x_{n-1}\right\rangle=0$ ?

The corresponding Freiheitssatz was proved by A. I. Shirshov 19 for free Lie algebras and by L. Makar-Limanov 12 for free associative algebras over a field of characteristic 0 .

If $L$ is a finitely presented Lie algebra with an undecidable word problem [2], then $P(L)$ is a finitely presented Poisson algebra with an undecidable word problem. Thus the word problem is undecidable for Poisson algebras in general formulation. The decidability of the word problem for Lie algebras with a single defining relation was also proved by A. I. Shirshov [19]. The analogs of this question for associative algebras and for semigroups are open. 
Problem 2. Is the word problem decidable for Poisson algebras with a single defining relation?

A set of elements $S$ of a free Lie (associative or Poisson) algebra is called free if the subalgebra generated by $S$ is free and $S$ is a free set of generators of this subalgebra.

It is well known 4 that elements $f$ and $g$ of a free associative algebra are not free if and only if $[f, g]=0$.

Problem 3. If $F$ is a field of characteristic 0 , then is it true that elements $f$ and $g$ of $P\left\langle x_{1}, x_{2}, \ldots, x_{n}\right\rangle$ are not free if and only if $[f, g]=0$ ?

Note that freeness and algebraic independence of elements are equivalent in the case of polynomial algebras. It is well known that the algebraic dependence of a finite set of elements of a polynomial algebra is algorithmically recognizable. There exists an algorithm which decides whether a finite set of elements in a free Lie algebra is free (see, for example [18, or [22]). It is also known that freeness of a finite set of elements is algorithmically unrecognizable for free associative algebras 20.

Problem 4. Is freeness of a finite set of elements of a free Poisson algebra algorithmically recognizable?

In 1968 P. Cohn 3 proved that the automorphisms of a free Lie algebra with a finite set of generators are tame. It is well known [5, 8, 10, 11 that automorphisms of polynomial algebras and free associative algebras in two variables are also tame.

Problem 5. Is it true that automorphisms of two-generated free Poisson algebras are tame?

Note that the Nagata automorphism is a wild automorphism of a free Poisson algebra in three variables.

\section{ACKNOWLEDGMENTS}

We are grateful to Max-Planck Institute für Mathematik for their hospitality and excellent working conditions.

\section{REFERENCES}

[1] G. M. Bergman, Centralizers in free associative algebras, Trans. Amer. Math. Soc., 137 (1969), 327-344. MR0236208 (38:4506)

[2] L. A. Bokut, Unsolvability of the word problem, and subalgebras of finitely presented Lie algebras, Izv. Akad. Nauk SSSR, Ser. Mat., 36 (1972), 1173-1219. MR0330250 (48:8588)

[3] P. M. Cohn, Subalgebras of free associative algebras, Proc. London Math. Soc., 56 (1964), 618-632. MR0167504 (29:4777)

[4] P. M. Cohn, Free rings and their relations, 2nd ed., Academic Press, London, 1985. MR0800091 (87e:16006)

[5] A. G. Czerniakiewicz, Automorphisms of a free associative algebra of rank 2, I, II, Trans. Amer. Math. Soc., 160 (1971), 393-401; 171 (1972), 309-315. MR0280549 (43:6269). MR0310021(46:9124)

[6] J. Donin and L. Makar-Limanov, Quantization of quadratic Poisson brackets on a polynomial algebra of three variables, J. Pure Appl. Algebra, 129 (1998), no. 3, 247-261. MR1631249 (99e:16036)

[7] I. M. Gelfand, A. A. Kirillov, Sur les corps liés aux algèbres enveloppantes des algèbres de Lie. (French) Inst. Hautes Études Sci. Publ. Math. No. 31, 1966, 5-19. MR0207918 (34:7731) 
[8] H. W. E. Jung, Uber ganze birationale Transformationen der Ebene, J. Reine Angew. Math., 184 (1942), 161-174. MR0008915 (5:74f)

[9] S. Kaliman, L. Makar-Limanov, On the Russel-Koras contractible threefolds, Journal of Algebraic Geometry, 6 (1997), 247-268. MR1489115 (98m:14041)

[10] W. van der Kulk, On polynomial rings in two variables, Nieuw Archief voor Wiskunde, (3)1 (1953), 33-41. MR0054574(14:941f)

[11] L. Makar-Limanov, The automorphisms of the free algebra with two generators, Funksional. Anal. i Prilozhen. 4 (1970), no. 3, 107-108; English translation: in Functional Anal. Appl. 4 (1970), 262-263. MR0271161 (42:6044)

[12] L. Makar-Limanov, Algebraically closed skew fields, J. Algebra, 93 (1985), no. 1, 117-135. MR0780486 (86d:16028)

[13] M. Nagata, On the automorphism group of $k[x, y]$, Lect. in Math., Kyoto Univ., Kinokuniya, Tokio, 1972. MR0337962 (49:2731)

[14] I. P. Shestakov, Quantization of Poisson superalgebras and speciality of Jordan Poisson superalgebras, Algebra i Logika, 32 (1993), no. 5, 571-584; English translation: in Algebra and Logic, 32 (1993), no. 5, 309-317. MR.1287006 (95c:17034)

[15] I. P. Shestakov and U. U. Umirbaev, The Nagata automorphism is wild, Proc. Nat. Acad. Sci. USA, 100 (2003), no. 22, 12561-12563. MR2017754 (2004j:13036)

[16] I. P. Shestakov and U. U. Umirbaev, Poisson brackets and two generated subalgebras of rings of polynomials, Journal of the American Mathematical Society, 17 (2004), 181-196. MR2015333 (2004k:13036)

[17] I. P. Shestakov and U. U. Umirbaev, Tame and wild automorphisms of rings of polynomials in three variables, Journal of the American Mathematical Society, 17 (2004), 197-227. MR2015334 (2004h:13022)

[18] A.I.Shirshov, Subalgebras of free Lie algebras, Mat. Sbornik, 33(75) (1953), 441-452. MR0059892 (15:596d)

[19] A. I. Shirshov, Some algorithm problems for Lie algebras, Sibirsk. Mat. Z., 3 (1962), 292-296. MR0183753 (32:1231)

[20] U. U. Umirbaev, Some algorithmic questions concerning associative algebras. (Russian) Algebra i Logika 32 (1993), no. 4, 450-470, 474; English translation: in Algebra and Logic, 32 (1993), no. 4, 244-255 (1994). MR1286789 (95e:03128)

[21] U. U. Umirbaev and I. P. Shestakov, Subalgebras and automorphisms of polynomial rings, Dokl. Akad. Nauk 386 (2002), no. 6, 745-748. MR2004473(2004i:14068)

[22] E. Witt, Die Unterringe der freien Lieschen Ringe, Math. Z., 64 (1956), 195-216. MR0077525 $(17: 1050 \mathrm{a})$

[23] A. Zaks, Dedekind subrings of $k\left[x_{1}, x_{2}, \ldots, x_{n}\right]$ are rings of polynomials, Israel J. Math., 9 (1971), 285-289. MR0280471 (43:6191)

Department of Mathematics \& Computer Science, Bar-Ilan University, 52900 RamatGan, Israel - and - Department of Mathematics, Wayne State University, Detroit, MiCHIGAN 48202

E-mail address: lml@math.wayne.edu

Department of Mathematics, Eurasian National University, Astana, 010008, KazaKHSTAN

E-mail address: umirbaev@yahoo.com 